Aus der Chirurgischen Abteilung des Städtischen Krankenhauses St. Georg in Leipzig. (Leitender Arzt: Prof. Heller.)

\title{
Erfahrungen mit Bluttransfusionen nach Oehlecker am chirurgischen Material.
}

Von Dr. Erich Hempel, Assistent an der Abteilung.

I.

Die Frage der Bluttransfusion ist in ein neues Stadium getreten. Es ist ein Verdienst Oehleckers, uns ein Verfahren angegeben zu haben, mittels dessen jeder chirurgisch und nicht chirurgisch Geschulte zuverlässig die gewünschte Blutmenge von einem Spender direkt zum Empfänger überleiten kann. Damit ist die techntische Frage, die allen, die sich damit beschäftigt haben, bisher immer wieder Schwierigkeiten bereitet hat, nun endgültig gelöst. Sache der Erörterung kann heute nur noch sein: Können wir von Bluttrans. fusionen überhaupt Nutzen erwarten, ohne den Empfänger irgend. einer Gefahr auszusêtzen, und in welchen Fällen sind Bluttransfusionen auszuführen?

An der Hand unseres Materials von Bluttransfusionen soll diese Frage kurz erörtert werden. Wir haben seit Frühjahr 1920 mit dem Oehleckerschen Apparat 31 Bluttransfusionen bei 26 Patienten ausgeführt. Gewil eine kleine Menge, im Vergleich zu den 150 Transfusionsfällen, die O e hlecker auf dem letzten Chirurgenkongre $B$ besprechen konnte. Wenn wir trotzdem unsere Erfahrungen damit, besonders nach Mißerfolgen in früheren Jahren mit anderen, unzulänglichen Methoden, hier veröffentlichen, so tun wir dies deshalb, weil wir den Eindruck gewonnen haben, daB das Oehleckersche Verfahreı noch immer nicht hinlänglich genug bekannt ist, und weil die Mitteilung guter Resultate auch von anderer Seite dem Verfahren und der Ållgemeinheit nur zugutekommen kann. Anschließend wollen wir zu den oben gestellten Fragen über Wertigkeit der Bluttrausfusion und Indikationen Stellung nehmen.

Die Transfusion nach Oehlecker ist eine direkte, von Vene zu Vene, mittels eines dazwischen geschalteten Zweiwegehahnes. Sie hat damit den Vorteil, das biologisch unverändertes Blut, unbeschädigte Blutkörperchen, unverändertes Blutplasma mit den verschiedensten Stoffen der Drüsen der inneren Sekretion übertragen werden. Daß die venöse Transfusion keine geringere Wirkung als die arterielle hat, geben alle Berichterstatter der Literatur zu. Es wăre auch nicht einzusehen, weshalb nicht, da sich die roten Blutkörperchen ja sehr bald in der Lunge mit Sauerstoff beladen. Durch eine Glasspritze ${ }^{1}$ ), die aul den Zweiwegehahn angeschlossen ist, wird aus der Vene des Spenders Blut angesaugt, der Zweiwegehahn zum Empfänger umgestellt und die Blutmenge in den venösen Kreislauf des Empfängers eingespritzt. Stand eine gut gefüllte Vena cubitalis mediana am gut gestauten Spenderarm nicht zur Verfügung, so nahmen wir in einigen Fällen, .ohne Schaden damit anzurichten, die tief liegende Hauptvene der Ellenbeuge. Durch eine neu angesetzte, mit Natr. citr. gefüllte gleiche Glasspritze wird das System kurz mit der gerinnungshemmenden Flüssigkeit ${ }^{2}$ ) gefüllt und erneut Blut angezogen und transfundiert.

Die Vorzüge des Oehleckerschen Verfahrens, die wir beobachtet haben, waren:

1. Einfachheit: Die Transfusion war unter allen äuBeren Umständen ausführbar (auch außerhalb unseres Krankenhauses). Jede

3) Die Spritzen mussen warm sein, damit keine Abklihlung des uberzuleitenden Blutes eintritt. Will man sie nicht in einem mit warmer Kochsalzlossung geftillten flachen Becken bei der Transfusion liegen haben, so soll man sie doch stets nach dem einzelnen Gebrauch mit warmer Kochsalzlösung ausspritzen, erstens, um sie warm zu erhalten, zweitens, um die Blutreste daraus zu entfernen und ihre Gleltfahigkelt zu erhalten. a) Im weiteren Verlauf der Transfusion benutzen wir jeizt dazu nur noch NaCl-Lobsung 
mit Asepsis und Umgang mit Instrumenten vertraute Kraft wird sie yornehmen körnen.

2. Zuverlässigkeit: Die Ueberleitung des Blutes glückte in allen Fällen. Jedesmal wurde die Transfusion nach freiem Willen beendet, niemals waren wir durch Blutgerinnung oder andere technische Zwischenfälle dazu gezwungen.

3. Dosierbarkeit: Man ist in der Lage, die Blutmenge wie ein Medikament zu dosieren, auch dem Spender vorauszusagen, wieviel Blut man ihm entnehmen will, soda $B$ er vor unerwünschten Verlusten geschützt ist. Diese Methode ermöglicht es außerdem, bei größeren Transfusionen dem Spender das verlorene Blutvolumen sofort im Anschluß an die Transfusion durch Ringersche Lösung $\mathrm{zu}$ ersetzen, soda $\beta$ also auch das Interesse des Spenders nach Möglichkeit gewahrt ist.

4. Das Tempo der Transfusion kann nach den besonderen Umständen bemessen werden. Im Anfang sahen wir die Raschheit der Ausführung als einen Vorteil dieser neuen Methode an. Sie ließ sich in 15-20 Minuten mit Leichtigkeit ausfähren. Jetzt erblicken wir darin keinen Vorteil mehr, halten sogar zu Anfang schnell ausgeführte Transfusionen unter Umständen für schädlich für den Emp-
fänger. Ich meine damit nicht die Ueberlastung des geschwächten rechten Herzens, von der manche Autoren ( $\mathrm{Z}$ III r) behauptet haben, da $B$ sie bei der venösen Transfusion einträte und leicht zum Herzstillstand führen könne. Wir haben eine solche stärkere, schadenstiftende Beanspruchung des venösen Kreislaufes (Blutstauung im rechten Herzen und in den Hohlvenen, Verhinderung des Abflusses des stark kohlensäurehaltigen Blutes aus den nervösen Zentralorganen, Asphyxie und plötzlicher Tod als Folge [Zeller]) auch nicht in Andeutungen wahrnehmen können. Aus anderen Gründen erscheint uns eine langsame, dosierbare Ueberleitung, die in Etappen ausgeführt wird, nötig. Da, wie wir noch sehen werden, die Voruntersuchungen auf Hämolyse und Agglutination uns keinen absolut sicheren Anhaltspunkt geben können, ob beide Blutarten sich auch in vivo vertragen, wenden wir gewisse VorsichtsmaBregeln an. Das ist erstens die prophylaktische Bluteinspritzung von $5-10 \mathrm{ccm}$. Diese biologische Probe ist schon von Abelman n angeraten worden, läßt sich aber nur mit dem Oehleckerschen Apparat einfach und sicher durchführen. Zweitens die klinische Beobachtung während der Transfusion selbst. Wir transfundieren zuerst $5-10 \mathrm{ccm}$, füllen das System mit Natr. citr. auf und warten ruhig $a b$, bis zu 5 Minuten, $\mathrm{ob}$ sich irgendwelche anaphylaktischen Erscheinungen, Beklemmung, Pulsbeschleunigung, Zyanose usw. einstellen. Wir haben mit anderen die Erfahrung gemacht, da $B$ die ersten Anzeichen der drohenden Hämolyse in wenigen Minuten nach Ueberleitung einer geringen Menge von Blut sich schon zeigen. Diese Probeüberleitung von Blut wiederholen wir ein zweites und drittes Mal, und dann erst gehen wir zu größeren Dosen allmählich steigend über. Gerade dieser Umstand, $\mathrm{da} B$ wir nach kleineren Probeinfusionen warten und dann langsam dem Körper das" neue Blut zuführen können, spricht für die Güte und Sicherheit dieser Methode. Niemals sahen wir die bei anderen Verfahren so leicht eintretende Gerinnung, obwohl wir bis zu 10 Minuten die Trausfusion unterbrochen haben. Thrombose und Emboliegefalır sind so gut wie ausgeschaltet.

Wir haben sogleich einige der wichtigsten VorsichtsmaBregeln, die vor und bei einer Transfusion anzuwenden sind, erwähnt. $\mathrm{DaB}$ der Spender frei von Syphilis (Wa.R.) und Tuberkulose sowie sonstigen Allgemeinkrankheiten sein muß, ist selbstverständlich. Oehlecker hat neuerdings auf einen Umstand noch hingewiesen, der sicherlich jetzt nach dem Krieg volle Beachtung verdient. Er erlebte bei einer Bluttransfusion eine Uebertragung von Malariaplasmodien, von einem Kriegsteilnehmer, der selbst nie malariakrank gewesen war und bei dem auch zur Zeit keine Plasmodien mehr festzustellen waren. Er erhebt daraus die berechtigte Forderung, daß jeder, der in einer Malariagegend war, gleich, ob er malariakrank gewesen oder nicht, vor Ablauf mehrerer. Jahre nicht als Blutspender in Betracht kommen darf.

Es ist nicht nötig, sämtliche Krankengeschichten unserer Fälle hier zu veröffentlichen. Ich will nur einige wenige aus dem interessanten Material herausgreifen und hier in extenso besprechen.

Die Bluttransfusion kam zur Anwendung:

1. Bei akutem Blutverlust: $2 \mathrm{mal}$ bei 2 Patienten: Bei septischen Blutungen aus der Art. obturatoria sin. bei Beckenosteomyelitis und Sepsis 1mal. Bei Arrosionsblutung aus der Art. femoris profunda bei chronischer Oberschenkelosteomyelitis nach Amputation $1 \mathrm{mal}$.

2. Bei sekundären Anämien: $29 \mathrm{mal}$ bei 24 Patienten. Bei blutenden Ulcera ventriculi als Vorbereitung zur Operation $3 \mathrm{mal}$ bei $3 \mathrm{~Pa}-$ tienten; bei Ulcera pept. jejuni $3 \mathrm{mal}$ bei 2 Patienten, als Vorbereitung zur Resektion $2 \mathrm{mal}$ bei 1 Patienten; nach der Rad.-Operation $1 \mathrm{mal}$ bei Ulcera ventriculi-Operationen zur Hebung des Allgemeinzustandes nach der Operation 3mal bei 3 Patienten; bei chronischen Verdauungsstörungen durch Darmadhtäsionen nach Adhäsions-Laparotomie $2 \mathrm{ma}$ bei 1 Patienten; bei malignem Abdominaltumor als Vorbereitung zur Operation 1mal bei 1 Patienten; bei entzündlichem Adnextumor posi partum als Vorbereitung zur Operation $2 \mathrm{mal}$ bei 1 Patienten; bei Lungenabszeß zur Hebung des Allgemeinzustandes nach der Operation $2 \mathrm{mal}$ bei 2 Patienten; bei Gallenblasenkarzinom und Cholämie als Vorbereitung zur Operation und zur Erhöhung der Blutgerinnbarkeit $2 \mathrm{mal}$ bei 1 Patienten; bei Nieren- und Darmblutungen bei hämorrhagischer Diathese $1 \mathrm{mal}$ bei 1 Patienten; bei paranephritischem AbszeB, post operativen Blutungen, zur Hebung des Allgemeinzustandes post operationem 1mal bei 1 Patienten; bei unklaren Darmblutungen Imal bei 1 Patienten; bei Pankreatitis und Fettgewebsnekrose zur Hebung des Allgemeinzustandes nach der Operation $1 \mathrm{mal}$ bei 1 Patienten; bei Carzinomi uteri et ventriculi zur Hebung des Allgemeinzustandes $1 \mathrm{mal}$ bei 1 Patienteri; bei Blaserimole zur Vorbereitung für die Uterusexstirpation Imal bei 1 Patienten; bei Hämorrhoiden mit stärkeren chronischell Blutungen vor Withead $1 \mathrm{mal}$ bei 1 Patienten; bei Aortitis luica, Endokarditis, Aneurysma der Art. poplitea zur Hebung des Allgemeinzustandes nach der Operation $2 \mathrm{mal}$ bei 1 Patienten; bei Knochen- und Gelenktuberkulose zur Hebung des Allgemeinzustandes $1 \mathrm{mal}$ bei 1 Patienten: bei Sepsis mit Leukopenie nach Angina $1 \mathrm{mal}$ bei 1 Patienten.

Man ersieht hieraus, daB wider Erwarten bei einem chirurgischen Material die Transfusion bei akutem Blutverlust seltener zar Anwendung gekommen ist, nur $2 \mathrm{mal}^{1}$ ), häufiger bei sekundären Anämien, entweder als Vorbereitung zur Operation (10mal hei 8 Patienten) oder als Unterstützung der Rekonvaleszenz nach der Operation (12mal bei 10 Patienten) oder zur Hebung des Allgemeinzustandes bei nicht operierteı Fällen ( $7 \mathrm{mal}$ bei 6 Patienten).

I. Bei den Transfusionen bei akuten Blutungen ist eine interessante Beobachtung $\mathrm{zu}$ verzeichnen, soda $B$ hier eine Krankengeschichte kurz angeführt werden soll ${ }^{2}$ ).

Fall 1: Fr. B., $12 \mathrm{jähriger} \mathrm{Knabe,} \mathrm{Sepsis,} \mathrm{Osteomyelitis} \mathrm{des}$ Beckenknochens mit Arrosion der Art. obturatoria sinistra.

Nahezu moribund eingeliefert: BewuBtlos, weite, nicht reagierende Pupillen, schnappende Atmung, kaum fühlbarer Puls, kalte Extremitäten. Spender: Vater, der erst von einer Blutüberleitung nichts wissen wollte, „da der Junge ja doch im Sterben sei $i^{\text {‘6 }}$

Voruntersuchung des Blutes in vitro und Blutbefund vor der Transfusion fehlt, da der Knabe sofort von der Aufnahme in das Operationshaus gebracht wurde, wo sogleich die Transfusion angestellt wurde.

Nach $500 \mathrm{ccm}$ Blutäbertragung war noch keine Aenderung zu verzeichnen. Nach $750 \mathrm{ccm}$ wurde die Atmung regelmäBiger und tief, der Puls besserte sich, der Junge wurde unruhig. Nach 1000,0 kehrte das BewuBtsein zurück, der Puls wurde voll, die Atmung ruhig, der Knabe war klar und nahm mit Interesse an den Vorgängen teil. Es trat keine Hämolyse auf. Die Besserung hielt eine Woche an, im Verlauf der 2. Woche steigerten sich die Erscheinungen der Sepsis durch die Beckenosteomyelitis, der der Knabe 2 Wochen nach der schweren Blutung und der Transfusion schließ. lich erlag.

Trotz des tödlichen Ausganges ist dieser Fall für die Rettung nach schwerstem Blutverlust ein außerordentlich eindrucksvoller $\mathbf{E r}$ folg, ein Erfolg, der auch bei schärfster. Kritik nur der Bluttrausfusion zugeschrieben werden kann. Dieser lehrreiche Fall zeigt uns, wie groß die Blutmenge ungefähr sein müßte, die wir einem stark aus. gebluteten Patienten überleiten möchten, um sein Leben zu er halten. $1000 \mathrm{ccm}$ waren bei einem 12jährigen Knaben notwendig, wir brauchen also für einen Erwachsenen noch größere Mengen. Diese werden wir kaum einem Spender zumuten können, wenn wir auch einmal in der Lage waren, einer kräftigen Person $1650 \mathrm{ccm}$ Blut zu entnehmen. Es stände an sich nichts im Wege, nach Beendigung der einen Transfusion den Spender aus dem Oehleckerschen System auszuschalten und einen neuen Spender einzuschalten. Technisch ließe sich das mit Leichtigkeit durchführen. Voraus. setzung wäre klinisch nur, daß gesunde Spender zur Verfügung stehen. Nur wird natürlich mit. Heranziehung eines neuen Blut. gebers die Gefahr der Hämolyse sich steigern und eine eventuelle durch den 2. Geber erzeugte Hämolyse das gute Resultat der ersten Transfusion völlig zunichtemachen. Man wird dieses Risikq nicht gern auf sich nehmen wollen, und ein solches bleibt es doch immer mit und ohne Voruntersuchung der beiden Blutarten, wie wir später noch sehen werden. Zugegeben, da $B$ diese Voruntersuchung uns auch bis zu einem gewissen Grade davor schützen kann, so sind wir doch in so eiligen Fällen kaum je in der Lage, sie anzustellen. Wir sind aber trotzdem berechtigt, in so dringenden Fällen, ohne diese eine Blutüberleitung zu wagen, besonders, wenn die oben angeführten VorsichtsmaBregeln beobachtet werden. Es wird aber auch gar nicht unbedingt notwendig sein, bei akutem Blutverlust die ganze verlorengegangene Blutmenge durch Blut $\mathrm{zu}$ ersetzen, wenn nur ungefähr wenigstens der normale Füllungszustand des Gefäßsystems mit möglichst normalem Blutdruck wieder hergestellt wird. Natürlich so viel als möglich mit Blut. Denn dieses enthält nun einmal die funktionstüchtigen Sauerstoffträger und lebenswichtigen Komplemente ${ }^{66}$ (Z elle r). Außerdem ist der Druck in den Blutgefäßen nach Bluttransfusionen längere Zeit erhöht als bei reinen Kochsalzinfusionen infolge geringerer Durchlässigkeit der

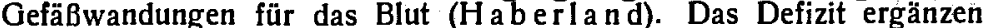
wir mit Ringerscher Lösung, die sich ja mit Leichtigkeit mittels des Oehleckerschen Apparates infundieren läBt. Es resultiert hieraus die Forderung, bei akuten Blutungen möglichst große Bluttransfusionen zu machen.

$\mathrm{DaB}$ bei akuten Blutungen die übertragene Blutmenge wenigstens einige Zeit funktionell eintreten kann, um den Körper über den gefährlichen Kollaps hinwegzubringen, beweisen experimentelle und klinische Beobachtungen auch anderer. Spenglers Versuche am ausgebluteten Hund und die Tierversuche $Z$ elle rs sind eine Stütze dafür. Wie lange die übertragene Blutmenge, Blutkörperchen und

1) Bei Extrauterin-Gravidităt wurde ausschlieblich die Autotransfusion nach $T$ h i e B : angewendet. - 2) Von Prof. Heller auf dem Chirurgenkongreb 1921 bereits erwathnt. 
Plasma, lebend 'im Empfänger bleiben, wann sie verschwinden, ist eine schwierige, noch nicht geklärte Frage. Wissen wir doch über die Dauer des Lebens der roten Blutkörperchen in unserer eigenen Blutbahn selbst noch nicht genügend Bescheid. Manche Autoren geben 1-2 Monate dafür an. Nach $\mathrm{Coe}$ ne n zerfallen die transfundierten roten Blutkörperchen am Ende der ersten Woche schon. Oehlecker hält 3-4 Wochen für die wahrscheinliche Lebensdauer. Er konnte keine Bilirubinzunahme im Blutserum beobachten, noch Veränderungen an den Erythrozyten. v. Ziemssen und $\mathrm{Sei}$ fert konnten einige Tage nach ihren Bluttransfusionen ein allmäh. liches Sinken -der Erythrozytenzahl, dann vom 4. Tage ein Ansteigen beobachten. Nach ihnen findet ein langsames, teilweises Zugrundegehen der roten Blutkörperchen statt, ohne Freiwerden größerer Mengen von Blutfarbstoff. Wir haben in der Reihe der Bluttransfusionen bei sekundären Anämien, die reaktionslos veřtragen wurden, einige Fälle längere Zeit durchgezählt und eine dauernde Gleich. mäBigkeit des Blutbefundes, sowohl zahlenmäBig als auch den Formen nach, feststellen können. Niemals beobachteten wir Erythrozytenschatten. Wir haben auch im Harn weder chemisch noch spektroskopisch Bilirubin oder Urobilin finden können, welches doch bei einem reichlichen Zugrundegehen von Erythrozyten hätte auftreten müssen. Selbst im Blutserum konnte in diesen Fällen bei regel. mäßiger Untersuchung keine Vermehrung des Bilirubingehaltes festgestellt werden. Unsere Zählungen erstreckten sich bis 3 Wochen nach der Transfusion. Wir wollen damit nicht behaupten, daß die übertragene Blutmenge in jedem Falle so lange Zeit die lebensunterhaltende Kraft darstellt. Ausgiebige Blutkörperchenzählungen, die das Weiterleben des transfundierten Blutes für eine gewisse Zeit beweisen, liegen von Hotz, von Vie rodt, Wederhake und $S$ i egel vor. Aber ein so langes Ueberleben wäre bei akuten Blutungen gar nicht einmal nötig. Wir wissen, daß sich bei akuten schwersten Blutverlusten die Blutmenge viel schneller ersetzt als bei chronischen geringfügigen Verlusteri. Selbst wenn die übertragenen roten Blutkörperchen als Sauerstoffträger nur wenige Tage überleben würden, würde dies für den rettenden Erfolg nach Bluttransfusionen bei akutem, schwerem Blutverlust genügen.

Ungezwungen ergibt sich bei den theoretischen Betrachtungen über den Wert der.Bluttransfusion ein Vergleich mit den autoplastischen und homoioplastischen Organtransplantationen. Wir wissen ja jetzt zur Genüge, daB nur autoplastische Transplantationen unter günstigsten Umständen Dauerbestand haben, daß homoioplastische Transplantationen mit wenigen, noch nicht sicher bewiesenen Ausnahmen zugrundegehen, aber wenigstens einige Zeit am Leben bleiben können. Es wäre merkwürdig, wenn es bei der Bluttransfusion anders wäre, bei der ja alle günstigen Bedingungen des Zellebens in einer Weise verwirklicht sind, wie sie bei keiner anderen Transplantationsform auch nur annähernd sich erreichen läßt

II. Bei den sekundären Anämien würde schon von einem Erfolg zu sprechen sein, wenn das übertragene Blut als Anreiz für die Blutneubildung wirkt, und dies scheint mit einer gewissen Regelmäßigkeit der Fall zu sein. Aus der größeren Zahl der Bluttransfusionen bei sekundären Anämien soll dafür eine weitere Krankengeschichte herangezogen werden.

Fall 2. Fr. V., 27 jähriger Mann. Ulcus ventriculi, Perforationsperitonitis. Naht + G. E. r. p., Spülung, Drainage.

Bluttransfusion 3 Monate später wegen schlechten Allgemeinzustandes, Ulkusblutung trotz Lenhartz-Kur, Gewichtsabnahme. Spender: Bruder (Wa.R. negativ). Voruntersuchung der beiden Blutarten o. B. Blutbefund vor der Transfusion: Hämoglobin $30 \%$, Erythrozyten 1900000 , Leukozyten 3500 . Menge des transfundierten Blutes: $250 \mathrm{ccm}$. Nach der Transfusion, die gut vertragen wurde, wesentlich frischeres, gesünderes Aussehen, subjektiv besseres Befinden. Blutbefund 24 Stunden nach der Transfusion: Hämoglobin $35 \%$, Erythrozyten 4200000 . Blutbefund 11/2 Monat nach der Transfusion: Hämoglobin $60 \%$, Erythrozyten 3700000 . Okkulte Blutungen stehen, anhaltende Gewichtszunahme, in 8 Wochen 27 Pfund.

Es trat bei diesem Patienten ein völliger Umschlag des Krank. heitsbildes ein, und zwar unmittelbar nach der Transfusion, soda $\beta$ sie nur durch diese zu erklären ist und nicht durch die allmähliche Erholung des Patienten infolge der weiter durchgeführten Lenhartz-Kur oder andere Zufälligkeiten. Die einmalige Transfusion von $250,0 \mathrm{ccm}$ war als Reizdosis gedacht, und als solche hat sie auch prompt gewirkt. Da wir in der Lage sind, bei dem. Oehleckerschen Apparat genau die Menge anzugeben, die wir überleiten, können wir bel der Kenntnis der Erythrozytenzahl in $1 \mathrm{cmm}$ des Spenderblutes genau berechnen, wieviel Erythrozyten wir überleiten, um wieviel also die Zahl der Erythrozyten beim Empfänger in $1 \mathrm{cmm}$ nach der Transfusion vermehrt sein müßte, wenn wir durch die Transfusion nur die Vermehrung des Blutes beim Empfänger um eine gewisse Menge erzielen wollten und nichts weiter. Uns ist aber bei allen Bluttransfusionen aufgefallen, daB diese Rechnung nicht stimmt, daß die Zahl der Erythrozyten nach der Transfusion bei genauester wiederholter Berechnung um vieles höher war, als sie nach der Menge des übergeleiteten Blutes $\mathrm{zu}$ erwarten war. Die Steigerung der Erythrozytenzahil von 1900000 auf 4200000 ist durch die übergeleitete Blutmenge von $250,0 \mathrm{ccm}$ allein nicht zu erklären. Wir dürfen diese Tatsachen so erklären, daß durch das übertragene Blut selbst oder die durch seinen Abbau entstandenen Produkte in den BlutkörperchenBildungsstätten des Empfängers alle halbreifen Formen, die des Uebergangs in die Blutbahn harren, auf einmal ins Blut ausgeschwemm1 werden, sodaß diese auffallende Erhöhung der Erythrozytenzahl plötz- lich eintritt. Es ist hierdur'ch also mathematisch der Beweis erbracht, daB eine Bluttransfusion als Reizdosis wirkt. Klinisch wußten wir das ja schon längst aus der Steigerung der Erythrozytenzahl nach einmaliger Injektion kleinerer Blutmengen.

Wăhrend wir bei der Bluttransfusion nach akutem Blutverlust nicht in der Lage sind, eine serologische Voruntersuchung auf Verträglichkeit beider Blutarten miteinander vorzunehmen, wegen der Dringlichkeit der Fälle, wird eine solche bei Bluttransfusionen bei allen anderen Fällen von den meisten ( $\mathrm{Schulz}, \mathrm{Hanssen,} \mathrm{Ka}$ liski) gefordert, wobei sich allerdings eine große Zahl von Beobachtern über die Unsicherheit der Reaktion im klaren ist (Crile, Pleh n, Umber usw.). Es ist vielfach beobachtet worden, daß in vitro diese Probe negativ ausfiel, und doch trat in vivo Hämolyse auf. Einzelne legen deshalb keinen Wert mehr auf diese Voruntersuchung. Für die Frage der Hämolyse und Agglutination liefern einige Krankengeschichten interessante Belege.

Fall 3. E. H., $32 \mathrm{jäh}$ rig e Frau. Postoperative Adhäsions. beschwerden. Sekundäre Anämie bei chronischen Verdauungsstörungen.

1. Spender der Bruder. Voruntersuchung und Blutbefund vor der Transfusion fehlt 1 ). Menge: $550,0 \mathrm{ccm}$. Gleich nach der Transfusion, bei der Patientin über Kopfschnierzen klagte und sehr unruhig war, traten schwere Störungen im Allgemeinbefinden auf, Kopfschmerzen Pulsbeschleunigung, Hämoglobinurie, Hämaturie, hämolytischer Ikterus. Nur langsam gingen diese Erscheinungen zurück. 8 Tage lang waren Erythrozyten in Harn nachweisbar, der Ikterus hielt 14 Tage an. Blutbefund 14 Tage nach der Transfusion: Hämoglobin 47\%, Erythrozyter1 - 4780000 , Leukozyten 5700 . Die nachträglich, 3 Wochen nach der Transfusion, vorgenommene serologische Blutuntersuchung ergab: Hämolyse des Empfängerblutes durch das des Spenders nicht eingetreten, dagegen starke Agglutination des Empfängerblutes durch das des Spenders $(++++$ mit 0,02 Serum). Außerdem wurde das Blut der Patientin mit artfremdem Blut, das im Laboratorium zur Wa.R. vorhanden war, zusammengebracht, dabei trat keine Hämolyse auf. Blutbefund 1 Monat nach der Transfusion: Hämoglobin 73\%, Erythrozyten 5000 000, Leukozyten 7200. Allgemeinbefinden etwas gebessert. 2 Monate später war eine 2 . Bluttransfusion auf $W$ unsch der Patientin vom Schwager geplant, unterblieb jedoch, da das Serum des Spenders gegenüber den gewaschenen Blutkörperchen des Empfängers geringe Autohämolysine bis zur Verdünnung 1:4 hatte, desgleichen umgekehrt sehr geringe Autohämolysine bis zur Verdünnung $1: 2$. 1 Monat später, also 3 Monate nach der ersten Transfusion, brachte die Patientin eine andere Patientin, eine Rekonvaleszentin auf der gleichen Station, die sich zu einer Bluttransfusion bereit erklärt hatte, und bat dringend um eine neue Bluttransfusion.

2. Spender: 17 jährige Patientin (Wa.R. negativ). Voruntersuchung: Empfänger: Autohämolysine gegen Blut der Spenderin in Spuren bis zur Serumverdünnung $1 / 2$ nachweisbar. Spender: Autohämolysine gegen. Blut in Spur bis zur Serumverdünnung $1 / 2$ nachweisbar. Trotzdem leichte Autohämolyse stattgefunden hatte, wird auf ausdrücklichen Wunsch der Patientin, die auf die Gefahren aufmerksam gemacht worden war, die Bluttransfusion vorgenommen. Blutbefund vor der Transfusion: Hämoglobin $80 \%$, Erythrozyten 4400000 , Leukozyten. 4800. Bei der Transfusion keine anaphylaktischen Symptome. Deslialb wurde die Blutmenge von $300 \mathrm{ccm}$ langsam übertragen. Blutbefund 1 Stunde nach der Transfusion: Hämoglobin $85 \%$, Erythrozyten 4600000 , Leukozyten 6700 . Blutbefund 24 Stunden nach der Transfusion: Hämoglobin 87\%, Erythrozyten 7200000 , Leukozyten 5000

Fall 4. E. Oe., 44 jähriger Mann, sekundäre Anämie nach Lungenabszeß operiert, der hier nur auszugsweise angeführt werden kann, verlief ähnlich.

Die Voruntersuchung hatte ergeben: Empfängerblutkörperchen gegen Serum der Spenderin 'Hämolyse + . Die Transfusion, die versehentlich angestellt worden war $(510,0 \mathrm{ccm})$, verlief ohne Besonderheiten, im Harn weder Bilirubin noch Urobilin nachweisbar. Keine Erythrozytenschatten, noch Erythrozyten, auch im Blutserum keine Bilirubinvermehrung, spektroskopisch: o. B. (Ein zweiter [Schluß-] Artikel folgt.) 